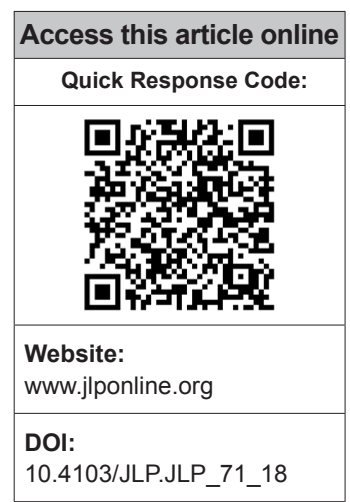

Department of Microbiology, Jagadguru Sri Shivarathreeshwara Medical College, Mysore, Karnataka, India

Address for correspondence: Dr. Visakha Kadiyala, Department of Microbiology, Jagadguru Sri Shivarathreshwara Medical College, SS Nagar, Bannimantap, Mysore - 570 015, Karnataka, India E-mail: visakhamicro2015 @gmail.com

Submission: 25-05-2018 Accepted: 23-09-2018

\section{Catheter associated urinary tract infections due to Trichosporon asahii}

\author{
Tejashree Anantharaj Urs, Visakha Kadiyala, Saundarya Deepak, Krishna Karthik M
}

\title{
Abstract:
}

Urinary tract infections (UTIs) caused by fungi, frequently associated with medical devices, have increased and caused great morbidity and mortality among hospitalized patients. Difficulties on different species identification as well as the lack of standardized sensitivity tests in vitro, contribute to the limited information available on epidemiology, diagnosis, and therapeutics of Trichosporon infections. There are only sporadic reports of UTI caused by Trichosporon asahii reported from India. We report six cases of UTI caused by $T$. asahii in severely ill patients in a tertiary care setup. Among six positive $T$. asahii UTI, four were found in female patients with a mean age of 60 years. We observed that all patients were on indwelling urinary catheter, broad-spectrum antibiotics, and with other comorbid conditions. With regard to the antifungal susceptibility testing, all the isolates were resistant to amphotericin B and sensitive to voriconazole. Majority of them were sensitive to Itraconazole, half of them were sensitive to fluconazole. The ubiquity and biofilm formation poses difficulty in establishing pathogenicity and delineating environmental or nosocomial infections. Risk factors such as use of antibiotics, indwelling catheter, and comorbidities such as hypertension, diabetes, anemia, and chronic kidney disease predispose for the development of UTI by T. asahii. Isolation of the same yeast in three consecutive urine samples with significant counts, along with significant number of pus cells establishes $T$. asahii as an etiological agent of UTI. Furthermore, the clearance of the fungus from the urinary tract with the recovery of the patient following catheter removal and antifungal therapy further confirms $T$. asahii as the cause of UTI.

Key words:

Nosocomial urinary tract infections, Trichosporon asahii, UTI's

\section{Introduction}

$\mathrm{U}$ rinary tract infection (UTI) is said "nosocomial" or "nosocomially acquired" (NUTI or NAUTI) when it is acquired in any health-care institution or, more generally, when it is related to patient management. ${ }^{[1]}$ Nosocomial UTIs account for $37.5 \%$ of all Hospital-acquired infections. ${ }^{[2]}$ With regard to the etiological agents of UTIs, bacterial species are prevalent, particularly among patients who use urinary catheters. ${ }^{[3]}$ UTIs caused by fungi, frequently associated with medical devices, have increased and caused great morbidity and mortality among nosocomial patients. ${ }^{[4]}$

This is an open access journal, and articles are distributed under the terms of the Creative Commons Attribution-NonCommercial-ShareAlike 4.0 License, which allows others to remix, tweak, and build upon the work non-commercially, as long as appropriate credit is given and the new creations are licensed under the identical terms.

For reprints contact: reprints@medknow.com
Although Candida albicans is still being isolated predominantly, the prevalence of Trichosporon has increased greatly. ${ }^{[5]}$ Genus Trichosporon is considered emerging pathogens in hospitalized patients. ${ }^{[6]}$ There are only sporadic reports of UTI caused by Trichosporon asahii reported from India.

Trichosporon species are widely distributed in nature and can occasionally belong to the human microbiota. They cause white piedra and onychomycosis in immunocompetent host as well as various localized and disseminated invasive infections in immunodeficient hosts. Virulence factors for trichosporonosis include glucuronoxylomannan in cell wall, proteases, phospholipases, and the ability to form biofilms. They form true mycelia,

How to cite this article: Urs TA, Kadiyala V, Deepak S, Karthik MK. Catheter associated urinary tract infections due to Trichosporon asahii. J Lab Physicians 2018;10:464-70. 
blastoconidia, and arthroconidia. Virulence factors and morphological structures may be exhibited differently in different species. Their ubiquity and biofilm formation may create confusion between colonized and truly infected patients. Invasive trichosporonosis may be confused with disseminated candidiasis. Although invasive trichosporonosis has been studied; however, there are no specific guidelines for clinical interpretation of Trichosporon recovery in urine. ${ }^{[7]}$ Azotemia and aggravation of renal dysfunction leading to renal failure may rarely occur. ${ }^{[8]}$

At present, there are 51 species of Trichosporon, 16 of which are able to cause human infections. T. asahii is the predominant species involved in systemic mycosis. ${ }^{[9]}$ The emergence of less common, but medically important opportunistic fungal pathogens has contributed to an increase in the rate of morbidity and mortality. ${ }^{[10,11]}$

We report six cases of UTI caused by T. asahii in severely ill patients in a tertiary care setup.

\section{Case Reports}

\section{Case 1}

A 68-year-old diabetic and hypertensive female patient was admitted to the hospital with chronic kidney disease and referred for Double J-stent insertion. She was a known case of chronic kidney disease and ischemic heart disease. Her blood parameters were as follows: hemoglobin $=8 \mathrm{~g} / \mathrm{dl}$, total leukocyte count $=12,800 / \mathrm{ml}$, urea $=150 \mathrm{mg} / \mathrm{dl}$, creatinine $=3.9 \mathrm{mg} / \mathrm{dl}$, and random glucose $=127 \mathrm{mg} / \mathrm{dl}$.

During the stay in the hospital, the patient had an indwelling catheter. The catheter was inserted using sterile technique. She was treated with meropenem, ciprofloxacin along with antacids and multivitamins. After 5 days of stay, she developed UTI. She had pyrexia of $102^{\circ} \mathrm{F}$.

Patient's urine collected using universal precautions. Urine wet mount showed plenty of pus cells, 2-4 epithelial cells and budding yeast cells. The urine sample was inoculated with a standard loop on blood agar and Urichrom agar (Himedia Laboratories, India) and incubated at $37^{\circ} \mathrm{C}$. On blood agar tiny, white, dry, wrinkled colonies were observed [Figure 1], and on urichrom agar blue, dry, wrinkled colonies were observed [Figure 2] with a colony count of $>10^{5} \mathrm{cfu} / \mathrm{ml}$. The Gram stain of the colony revealed septate hyphae with arthrospores and few budding yeast cells [Figure 3]. The colony was subcultured on to Potato Dextrose Agar and incubated at $22^{\circ} \mathrm{C}$ and $37^{\circ} \mathrm{C}$. At both, the temperatures colonies of yeast-like fungus were obtained in pure culture. After 5 days of incubation on Corn Meal Agar (CMA), abundant rectangular arthroconidia were seen [Figure 4]. When the patient was on the same urinary catheter, two more samples were collected. Both the samples revealed the same picture. The yeast was identified as T. asahii with Gram stain, morphology on CMA, hydrolysis of urea, carbohydrate fermentation, and assimilation tests. The same was identified by Vitek 2 compact automated system (bioMeriux). For molecular identification, the isolate was sent to the National Culture Collection of Pathogenic Fungi (NCCPF), Department of Medical Microbiology, Postgraduate Institute of Medical Education and Research, Chandigarh, which confirmed our identification.

Antifungal susceptibility testing was done by using Mueller-Hinton Agar with 2\% Glucose and methylene blue (Himedia, India) for fluconazole, Itraconazole, voriconazole, Ketoconazole, and Amphotericin-B. The susceptibility of the strain was evaluated by the disk-diffusion method, in accordance with protocol M44-A of the clinical and laboratory standards institute. It was found that this isolate was sensitive to Itraconazole and voriconazole and resistant to fluconazole, Ketoconazole, and Amphotericin-B. Antifungal therapy with voriconazole and change of indwelling catheter subsequently lead to negative fungal growth in urine cultures.

\section{Case 2}

T. asahii was isolated thrice from urine samples of a 42-year-old comatose patient who was admitted for follow-up and rehabilitation. She was a known case of hypertension and hypothyroidism. She underwent a surgery for left temporal, parietal intracranial bleed with IV extension. Her blood parameters were as follows, hemoglobin $=10.7 \mathrm{~g} / \mathrm{dl}$, total leukocyte count $=21,100 / \mathrm{ml}$, urea $=81 \mathrm{mg} / \mathrm{dl}$, creatinine $=0.9 \mathrm{mg} / \mathrm{dl}$, and glucose random $=110 \mathrm{~g} / \mathrm{dl}$. The patient had an indwelling catheter throughout her stay in the hospital. The patient was on clindamycin, cefoperazone, levothyroxine, amlodipine, and antacids.

Urine microscopy revealed moderate number of pus cells and occasional yeast cells. The sample was subjected to culture, and pure, significant, white wrinkled colonies were isolated which showed true hyphae and arthroconidia on staining. The isolate was identifi ed as T. asahii based on Grams staining, morphology on CMA, urea hydrolysis [Figure 5], carbohydrate fermentation, and assimilation tests [Figure 6] and the same was confirmed by Vitek 2 compact automated system (Biomerieux).

Antifungal susceptibility testing revealed that the isolate was sensitive to Itraconazole, voriconazole and resistant to Amphotericin-B, fluconazole, and Ketoconazole [Figure 7]. Prompt treatment with voriconazole and 
management for comorbidities lead to subsequently sterile urine cultures.

\section{Case 3}

Urine sample of a 53-year-old female patient with colon carcinoma and bilateral hydroureteronephrosis yielded the growth of $T$. asahii on three occasions. The patient underwent hemicolectomy and was on chemotherapy. The patient had a urinary catheter throughout her stay in the hospital. The patient was on treatment with Amoxyclav, Linezolid, and Levofloxacin in appropriate dosages along with antacids and multivitamins. Her blood picture showed hemoglobin $=6.9 \mathrm{mg} / \mathrm{dl}$, total leukocyte count $=13,600 / \mathrm{ml}$, urea $=72 \mathrm{mg} / \mathrm{dl}$, and creatinine $=4.2 \mathrm{mg} / \mathrm{dl}$.

Wet mount of the urine sample revealed moderate number of pus cells and budding yeast cells with pseudohyphae [Figure 8]. Based on cultural and biochemical characteristics, the isolate was identified as T. asahii which is sensitive to voriconazole, fluconazole and resistant to Amphotericin-B, Itraconazole, and ketoconazole. Antifungal therapy with fluconazole was given, and urine sample sent for the culture after treatment which was found to be negative for the fungus.

\section{Case 4}

T. asahii was isolated thrice from urine samples of an 85-year-old male patient who was admitted for generalized weakness for 15 days' duration and was a known case of hypertension, diabetes mellitus, and asthma. Radiological investigations revealed right mild hydronephrosis with proximal ureteric calculus and left mild hydronephrosis with partial staghorn calculus. He was diagnosed as acute on chronic kidney failure and obstructive nephropathy. The patient had an indwelling catheter throughout his stay in the hospital. He was treated with ciprofloxacin, clindamycin, Amlong, Asthalin nebulization, insulin, and antacids. His blood parameters were as follows: hemoglobin $=12 \mathrm{~g} / \mathrm{dl}$, total leukocyte count $=16,500 / \mathrm{ml}$, urea $=280 \mathrm{mg} / \mathrm{dl}$, creatinine $=8 \mathrm{mg} / \mathrm{dl}$, and glucose random $=120 \mathrm{mg} / \mathrm{dl}$.

Urine microscopy revealed plenty of pus cells and budding yeast cells. Culture isolate was identified as $T$. asahii based on the cultural and biochemical characteristics which are sensitive to itraconazole, voriconazole and resistant to fluconazole, ketoconazole, and Amphotericin-B. The removal of urinary catheter, the management of comorbidities and treatment with voriconazole subsequently lead to sterile urine cultures.

\section{Case 5}

T. asahii was isolated from urine on three occasions from a 60-year-old male patient who was admitted with the history of fever and abdominal pain from 15 days. He underwent cholecystectomy and was on treatment with cefoperazone and clindamycin. He was on indwelling catheter for 6 days' duration. His blood parameters were as follows: hemoglobin $=13 \mathrm{~g} / \mathrm{dl}$, total leukocyte count $=12,000 / \mathrm{ml}$, urea $=81 \mathrm{mg} / \mathrm{dl}$, creatinine $=1.2 \mathrm{mg} / \mathrm{dl}$, and fasting blood sugar $=80 \mathrm{mg} / \mathrm{dl}$. Urine microscopy showed 10-12 pus cells/HPF and few budding yeast cells. Culture yielded pure yeasty colonies which on Gram stain revealed true hyphae and arthroconidia. Based on culture morphology, biochemical reactions, and Vitek 2 identification, the isolate was identified as T. asahii which was sensitive to voriconazole, Itraconazole, fluconazole and resistant to Amphotericin-B and ketoconazole. Antifungal therapy with fluconazole and removal of indwelling catheter subsequently lead to negative fungal growth in urine cultures.

\section{Case 6}

A 55-year-old female patient was involved in a road traffic accident and underwent right knee surgery with vancomycin and ciprofloxacin antibiotic coverage. She was on indwelling urinary catheter for 7 days' duration. Her blood picture showed hemoglobin $=13 \mathrm{~g} / \mathrm{dl}$, total leukocyte count $=13,700 / \mathrm{ml}$, fasting and postprandial blood sugars $90 \mathrm{mg} / \mathrm{dl}$ and $140 \mathrm{mg} / \mathrm{dl}$, respectively, urea $=40 \mathrm{mg} / \mathrm{dl}$ and creatinine $=0.9 \mathrm{mg} / \mathrm{dl}$. Urine sample was sent for culture and sensitivity. Urine microscopy revealed plenty of pus cells with septate hyphae and arthroconidia. The culture isolate was identified as T. asahii through its, cultural characteristics, morphology on CMA, hydrolysis of urea, carbohydrate fermentation, and assimilation tests. The isolate was sensitive to fluconazole, itraconazole, voriconazole and resistant to Amphotericin-B, ketoconazole. Two more repeat urine analysis could not be done as the patient was discharged and lost to follow-up [Table 1].

\section{Results}

Among six positive T. asahii UTI, four were found in female patients.

Positive cultures were more common among individuals $>50$ years.

All patients were on indwelling urinary catheter with a mean duration of 18.8 days.

All patients received antibiotic therapy before the fungal infection. The most commonly used antibiotics were Beta-lactam group of antibiotics and fluoroquinolones.

Of the 6 cases, the presence of comorbid conditions such as diabetes in $33 \%$, hypertension in $50 \%$, anemia in $50 \%$, and chronic kidney disease in 33\% were seen. 
Table 1: Underlying conditions and risk factors among the study participants

\begin{tabular}{|c|c|c|c|c|c|c|}
\hline \multirow[t]{2}{*}{ Age/sex } & \multirow[t]{2}{*}{ Underlying conditions } & \multicolumn{5}{|c|}{ Risk factors } \\
\hline & & $\begin{array}{c}\text { Duration of use of } \\
\text { indwelling catheter (days) }\end{array}$ & $\begin{array}{l}\text { Diabetes } \\
\text { mellitus }\end{array}$ & Hypertension & Anemia & $\begin{array}{c}\text { On } \\
\text { antibiotics }\end{array}$ \\
\hline $68 /$ female & CKD patient admitted for double $\mathrm{J}$ stenting & 16 & Yes & Yes & Yes & Yes \\
\hline 42/female & Postoperative neurosurgery patient & 32 & No & Yes & Yes & Yes \\
\hline 53/female & Colon cancer with bilateral hydroureteronephrosis & 25 & No & No & Yes & Yes \\
\hline $85 /$ male & Acute on chronic kidney failure & 27 & Yes & Yes & No & Yes \\
\hline $60 /$ male & Postoperative cholecystectomy patient & 6 & No & No & No & Yes \\
\hline 55/female & RTA case & 7 & No & No & No & Yes \\
\hline
\end{tabular}

CKD = Chronic kidney disease, RTA = Road traffic accident

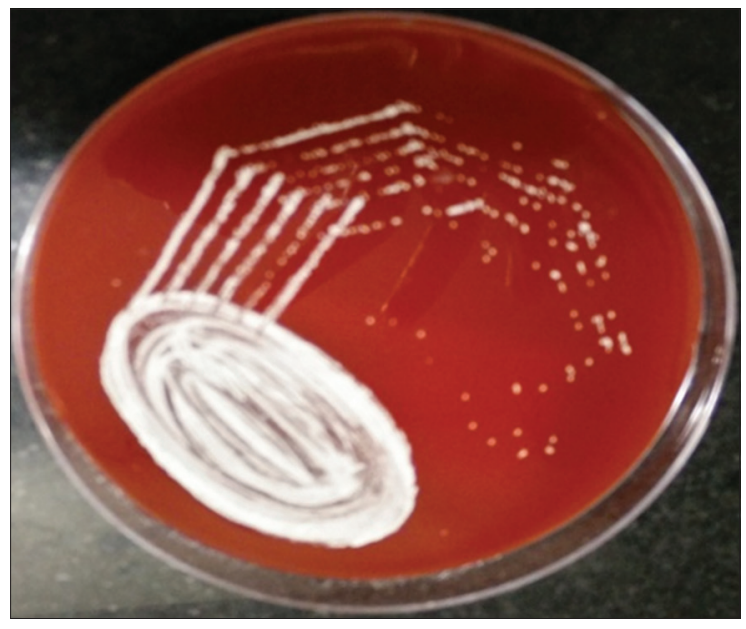

Figure 1: Tiny white, dry colonies on blood agar

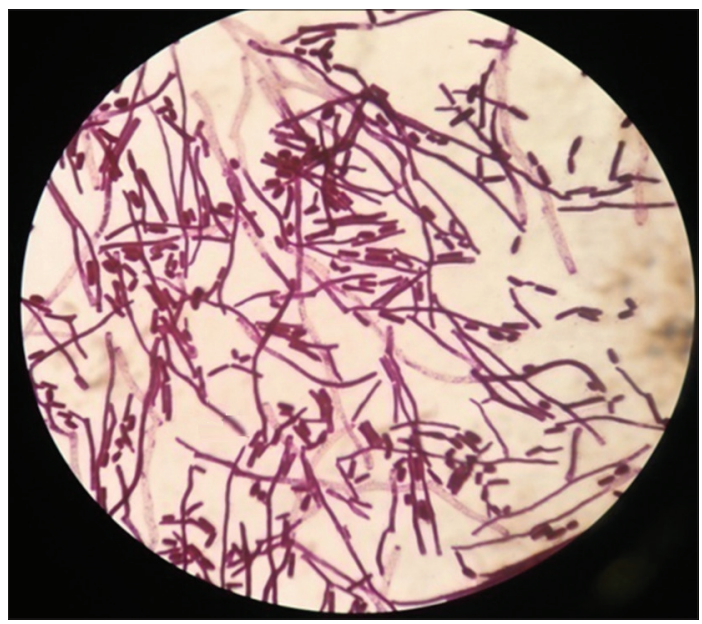

Figure 3: Grams stain of the colony showing hyphal forms with arthrospores and few budding yeast cells

With regard to the antifungal susceptibility testing, all the isolates were resistant to Amphotericin B and ketoconazole and sensitive to voriconazole. $83 \%$ of them were sensitive to Itraconazole, $50 \%$ of them were sensitive to fluconazole.

\section{Discussion}

Our results indicate that the prevalence of Trichosporon

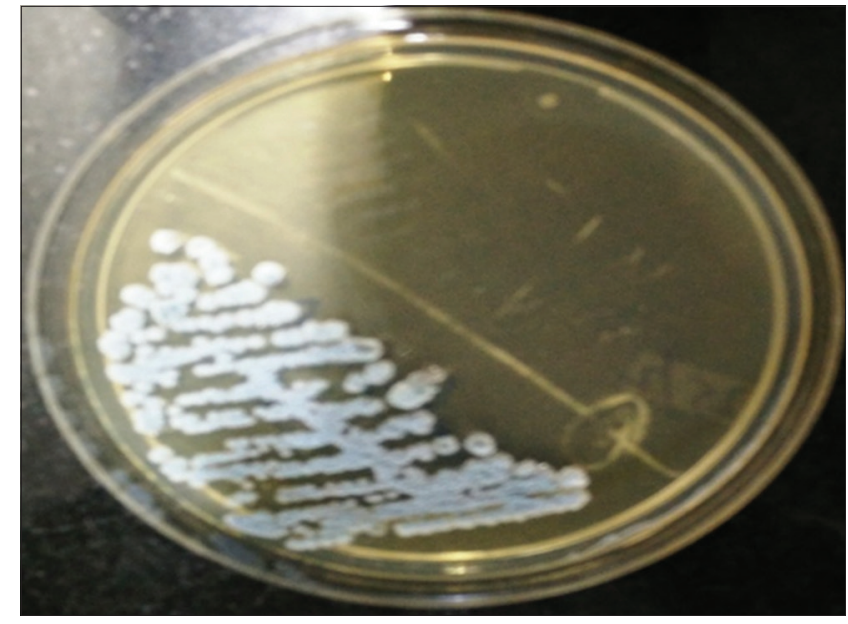

Figure 2: Blue, dry wrinkled, colonies on urichrom agar with a colony count of $>10^{5}$ $\mathrm{CFU} / \mathrm{ml}$ after $24 \mathrm{~h}$ incubation at $37^{\circ} \mathrm{C}$

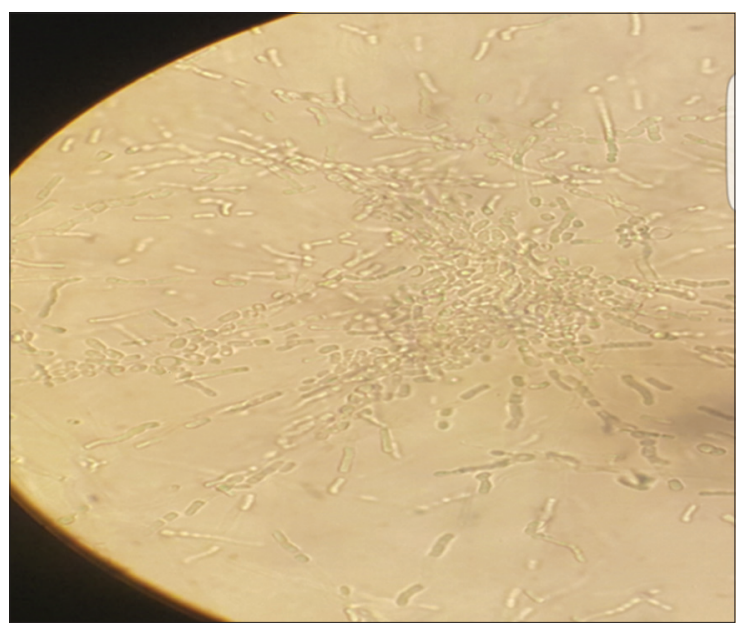

Figure 4: Corn meal agar showing pseudohyphae with blastoconidia of varying sizes and rectangular arthroconidia

spp. in urine cultures is more common among women compared to men with a mean age being 60 years. We observed that all patients were catheterized and were on broad-spectrum antibiotics and with other comorbid conditions.

In the present case series, a larger number of patients were of age group $>50$ years. These individuals could 


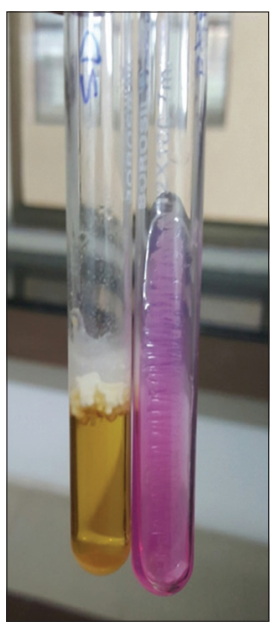

Figure 5: Sabourauds dextrose broth showing pellicle formation and urease test positive

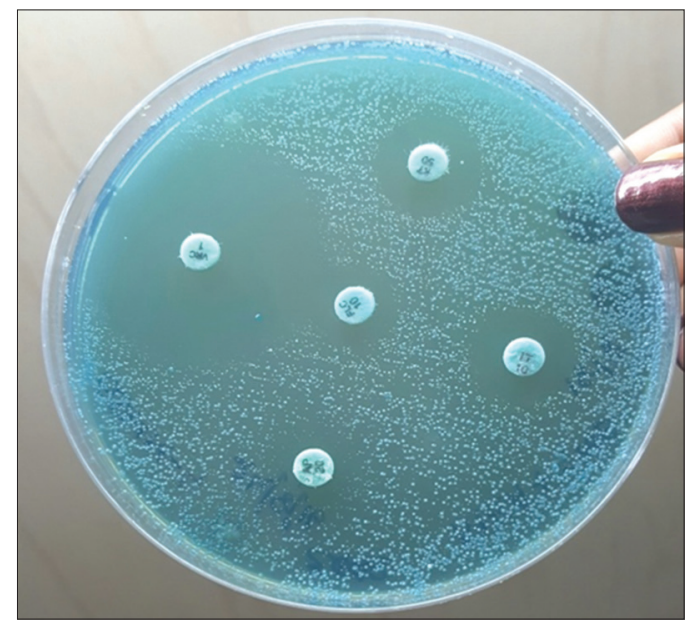

Figure 7: Antibiotic susceptibility testing on Mueller-Hinton Agar with $2 \%$ glucose and methylene blue showing semiconfluent growth of the test isolate

possibly have chronic conditions associated with immunosuppression and increased susceptibility to atypical urinary infections.

In general, urinary infections in adults are more common among women. This increased susceptibility is due to anatomical conditions, i.e., shorter urethra that is in close proximity to the vagina and anus. ${ }^{[12]}$ This finding was corroborated by this study as $66.6 \%$ of the infections occurred in women. In contrary, a study done in Brazil showed $65 \%$ of Trichosporon UTI occurred in men. ${ }^{[13]}$

Indwelling catheters compromises the natural barriers of the skin and mucosa, increasing the risk of opportunistic infections and complications, including UTIs. ${ }^{[14]}$ Fungal development among patients using indwelling catheters is facilitated by the formation of biofilm, which could explain the persistence of infection with Trichosporon spp. ${ }^{[15]}$ In our study, a similar finding was noted that all patients were on

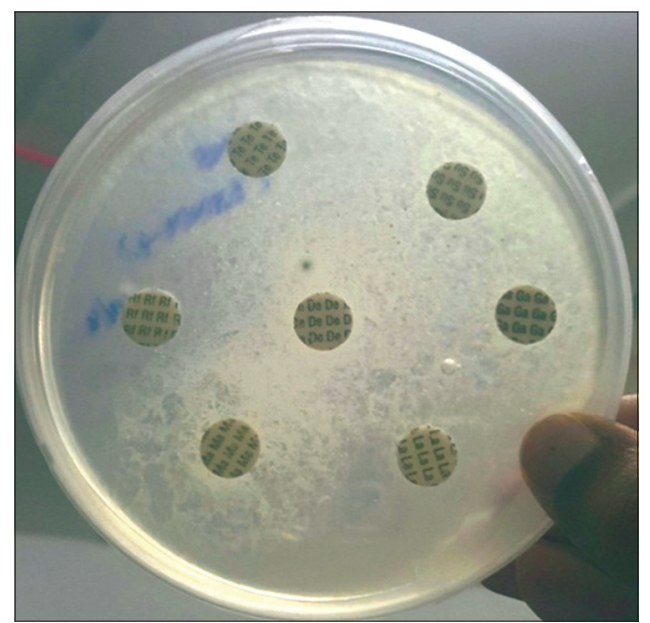

Figure 6: Sugar assimilation test showing assimilation of dextrose, maltose, sucrose, galactose, and lactose sugars

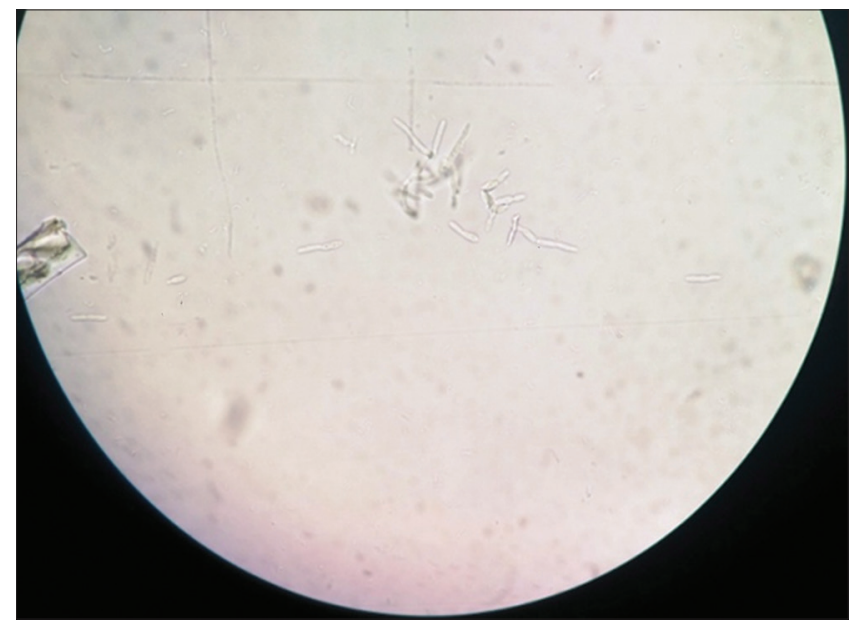

Figure 8: Wet mount of urine showing budding yeast cells, pseudohyphae and true hyphal forms

indwelling urinary catheter with a mean duration of 18.8 days. Multivariate analyses have emphasized that the duration of catheterization is the most important risk factor in the development of catheter-associated bacteriuria. ${ }^{[16-18]}$ Other risk factors include colonization of the drainage bag, catheter, and periurethral segment, diabetes mellitus, female gender, impaired renal function, poor quality of catheter care, including its insertion outside of an operating room and lack of antimicrobial therapy. ${ }^{[19-22]}$

The use of antibiotics in severely ill patients favors fungal development, particularly by yeasts, and the onset of opportunistic infections. Narrow and broad-spectrum antibiotics have broader mechanisms of action against bacteria when used in combination, and they selectively favor fungal growth in hospitalized patients. ${ }^{[23]}$ In our study, a relevant observation was seen that all patients used antibiotics, particularly cephalosporins and quinolones before infection. 
Consistent with previous studies conducted in Brazil and Spain, there were susceptible populations commonly associated with Trichosporon infections in this study. ${ }^{[7,24]}$

All six patients were severely ill and elderly ( $>50$ years), were on antibiotic treatment and indwelling urinary catheter. All patients had various comorbidities, among which Hypertension and Anemia were maximum followed by Diabetes and Chronic Kidney disease.

Data on T. asahii antifungal susceptibility are limited, but azoles have good activity against the fungus. While amphotericin $B$ has been shown to have a limited in vitro effect. ${ }^{[25,26]}$ The isolates in the present case series also showed resistance to Amphotericin B and were found variably sensitive to azoles (fluconazole, Itraconazole, and voriconazole). With concerns about the emergence of antifungal resistance, it is essential that prophylactic and empiric antifungal therapy be based on appropriately designed clinical studies, particularly in high-risk patients. Further studies are needed to define optimal approaches to facilitate earlier treatment, which will improve patient outcomes.

Difficulties on different species identification as well as the lack of standardized sensitivity tests in vitro, contribute to the limited information available on epidemiology, diagnosis, and therapeutics of Trichosporon infections.

Standardization of the approaches for early detection of this etiological agent is essential to ensure appropriate and effective treatment of severely ill patients. Standardization is required to differentiate between colonization and infection by Trichosporon spp., particularly among patients on urinary catheters as in the present case series.

The limitations of this study include the fact it was conducted in a single center and the small sample size.

\section{Conclusion}

Risks factors, such as presence of indwelling catheter, use of broad-spectrum antibiotics, and the presence of comorbid conditions such as diabetes mellitus, hypertension, chronic kidney disease, and anemia, predispose for the development of UTI by T. asahii.

Isolation of the same yeast in three consecutive urine samples with significant counts, along with a significant number of pus cells establishes T. asahii as an etiological agent of UTI. Furthermore, the clearance of the fungus from the urinary tract with the recovery of the patient following catheter removal and antifungal therapy further confirms T. asahii as the cause of UTI. ${ }^{[5]}$

\section{Declaration of patient consent}

The authors certify that they have obtained all appropriate patient consent forms. In the form the patient(s) has/ have given his/her/their consent for his/her/their images and other clinical information to be reported in the journal. The patients understand that their names and initials will not be published and due efforts will be made to conceal their identity, but anonymity cannot be guaranteed.

\section{Acknowledgment}

The erudite help and support by NCCPF, Department of Medical Microbiology, Postgraduate Institute of Medical Education and Research, Chandigarh, India, in the molecular identification of the first isolate is gratefully acknowledged.

\section{Financial support and sponsorship} Nil.

\section{Conflicts of interest}

There are no conflicts of interest.

\section{References}

1. Botto H. Nosocomial urinary tract infection (NUTI) in adult patients. Med Mal Infect 2003;33:216-7.

2. Ahoyo TA, Bankolé HS, Adéoti FM, Gbohoun AA, Assavèdo S, Amoussou-Guénou $\mathrm{M}$, et al. Prevalence of nosocomial infections and anti-infective therapy in Benin: Results of the first nationwide survey in 2012. Antimicrob Resist Infect Control 2014;3:17.

3. Lucchetti G, Silva AJ, Ueda SM, Perez MC, Mimica LM. Infections of urinary tract: analysis of frequency and sensitivity profile the causative agents of urinary tract infections in patients with chronic bladder catheterization. J Bras Patol Med Lab 2005;41:383-9.

4. Frank DN, Wilson SS, St Amand AL, Pace NR. Culture-independent microbiological analysis of Foley urinary catheter biofilms. PLoS One 2009;4:e7811.

5. Kumar S, Bandyopadhyay M, Mondal S, Pal N. A rare case of nosocomial urinary tract infection due to Trichosporon asahii. J Glob Infect Dis 2011;3:309-10.

6. Júnior AA, Carvalho RT, Focaccia R, Fernandez JG, Araújo HB, Strabelli TM, et al. Emergency of Trichosporon asahii infection in patients with heart failure in unit intensive care unit. Case report and review of literature. Rev Bras Ter Intensiva 2010;20:106-9.

7. Colombo AL, Padovan AC, Chaves GM. Current knowledge of Trichosporon spp. and trichosporonosis. Clin Microbiol Rev 2011;24:682-700.

8. Sood S, Pathak D, Sharma R, Rishi S. Urinary tract infection by Trichosporon asahii. Indian J Med Microbiol 2006;24:294-6.

9. Chander J. Miscellaneous oppurtunistic mycoses. Textbook of Medical Microbiology. $4^{\text {th }}$ ed., Ch. 28. New Delhi: Jaypee Brothers Medical Publishers Private Limited; 2018. p. 614.

10. Anaissie E. Opportunistic mycoses in the immunocompromised host: Experience at a cancer center and review. Clin Infect Dis 1992;14 Suppl 1:S43-53.

11. Girmenia C, Pagano L, Martino B, D'Antonio D, Fanci R, Specchia G, et al. Invasive infections caused by Trichosporon species and Geotrichum capitatum in patients with hematological malignancies: A retrospective multicenter study from Italy and review of the literature. J Clin Microbiol 2005;43:1818-28.

12. Lopes HV, Tavares W. Diagnosis of urinary tract infections. Rev 
Assoc Méd Bras 2005;51:306-8.

13. Mattede Md, Piras C, Mattede KD, Ferrari AT, Baldotto LS, Assbu MS, et al. Urinary tract infections due to Trichosporon spp. in severely ill patients in an Intensive Care Unit. Rev Bras Ter Intensiva 2015;27:247-51.

14. Abelha FJ, Castro MA, Landeiro NM, Neves AM, Santos CC. Mortality and length of stay in a therapy unit intensive surgical. Rev Bras Anestesiol 2006;56:34-45.

15. Khan ID, Sahni AK, Basu A, Haleem S. Trichosporon asahii urinary tract infection in immunocompetent patients. Med J Armed Forces India 2015;71:373-6.

16. Garibaldi RA, Burke JP, Britt MR, Miller MA, Smith CB. Meatal colonization and catheter-associated bacteriuria. N Engl J Med 1980;303:316-8.

17. Platt R, Polk BF, Murdock B, Rosner B. Risk factors for nosocomial urinary tract infection. Am J Epidemiol 1986;124:977-85.

18. Warren J, Bakke A, Desgranchamps F, Johnson JR, Kumon H, Shah J, et al. Catheter-Associated Bacteriuria and the Role of Biomaterial in Prevention. Nosocomial and Health Care Associated Infections in Urology. Plymouth, UK: Health Publications Ltd.; 2001. p. 153-76.

19. Sedor J, Mulholland SG. Hospital-acquired urinary tract infections associated with the indwelling catheter. Urol Clin North Am 1999;26:821-8.
20. Stamm WE, Hooton TM, Johnson JR, Johnson C, Stapleton A, Roberts PL, et al. Urinary tract infections: From pathogenesis to treatment. J Infect Dis 1989;159:400-6.

21. Maki DG, Tambyah PA. Engineering out the risk for infection with urinary catheters. Emerg Infect Dis 2001;7:342-7.

22. Tenke P, Kovacs B, Bjerklund Johansen TE, Matsumoto T, Tambyah PA, Naber KG, et al. European and Asian guidelines on management and prevention of catheter-associated urinary tract infections. Int J Antimicrob Agents 2008;31 Suppl 1:S68-78.

23. Menezes EA, Carneiro HM, Cunha FA, Oliveira IR, Ângelo MR, Salviano MN. Frequency of microorganisms causing hospital urinary tract infections in general hospital patients from Fortaleza. Rev Bras Anal Clin 2005;37:243-6.

24. Rodriguez-Tudela JL, Diaz-Guerra TM, Mellado E, Cano V, Tapia C, Perkins A, et al. Susceptibility patterns and molecular identification of Trichosporon species. Antimicrob Agents Chemother 2005;49:4026-34.

25. Walsh TJ, Melcher GP, Rinaldi MG, Lecciones J, McGough DA, Kelly $\mathrm{P}$, et al. Trichosporon beigelii, an emerging pathogen resistant to amphotericin B. J Clin Microbiol 1990;28:1616-22.

26. McGinnis MR, Pasarell L, Sutton DA, Fothergill AW, Cooper CR Jr., Rinaldi MG, et al. In vitro activity of voriconazole against selected fungi. Med Mycol 1998;36:239-42. 